\title{
SINGULARITIES AND RADICAL INITIAL IDEALS
}

\author{
ALEXANDRU CONSTANTINESCU, EMANUELA DE NEGRI, AND MATTEO VARBARO
}

\begin{abstract}
What kind of reduced monomial schemes can be obtained as a Gröbner degeneration of a smooth projective variety? Our conjectured answer is: only StanleyReisner schemes associated to acyclic Cohen-Macaulay simplicial complexes. This would imply, in particular, that only curves of genus zero have such a degeneration. We prove this conjecture for degrevlex orders, for elliptic curves over real number fields, for boundaries of cross-polytopes, and for leafless graphs. We discuss consequences for rational and F-rational singularities of algebras with straightening laws.
\end{abstract}

\section{INTRODUCTION}

A deformation of a scheme $X$ is a flat family $\mathcal{X} \longrightarrow \mathrm{T}$, over some connected affine scheme $T$, whose special fibre is $X$. Passing from an ideal $I$ of the polynomial ring $\mathbb{K}\left[x_{1}, \ldots, x_{n}\right]$ to its initial ideal gives rise to a flat family over the parameter space $\operatorname{Spec} \mathbb{K}[t]$ in which the special fibre (the one over $t=0$ ) corresponds to the initial ideal. We will call such a family a Gröbner deformation. When the generic fibre of the family is smooth, we call such a deformation a (Gröbner) smoothing. We focus our attention to the situation in which the special fibre is a Stanley-Reisner scheme. This means that $X$ is defined by a square-free monomial ideal $\mathrm{I}_{\Delta}$ corresponding to some simplicial complex $\Delta$. Specifically, we are looking at the interplay between the type of singularities (or the lack of such) in the generic fibre and the topology of the simplicial complex associated to the special fibre. We have two reasons for doing this. On the one hand, having a square-free initial ideal is a desirable property which better preserves homological invariants under degeneration [CV18]. For example, extremal Betti numbers stay constant (in value and position), and thus also depth and regularity, so Cohen-Macaulayness is passed on. Radical initial ideals appear also in toric settings, where, in certain cases, they admit a description in terms of unimodular triangulations of lattice polytopes [Stu96, BGT97, BR07, HP09]. On the other hand, by upper semi-continuity, smoothings (when possible) of Stanley-Reisner schemes associated to combinatorial manifolds produce important algebraic varieties: spheres smoothen to Calabi-Yau's, tori to Abelian varieties, and triangulations of $\mathbb{R} \mathbb{P}^{2}$ smoothen to Enriques surfaces. Studies in this direction have been done in [AC04, AC10, Chr11, CI14, IT17].

Throughout this introduction, the polynomial ring $\mathbb{K}\left[x_{1}, \ldots, x_{n}\right]$ will always be equipped with the standard $\mathbb{Z}$-grading given by $\operatorname{deg} x_{i}=1$. The two reasons presented above are reflected by two approaches. In the first one, where the generic fibre is fixed, we start our investigation by merging two similar questions, [Var19, Problem 3.6] and [CV18, Question 4.2], into one conjecture.

Conjecture 1. Let $\mathrm{I} \subset \mathbb{K}\left[\mathrm{x}_{1}, \ldots, \mathrm{x}_{\mathrm{n}}\right]$ be a homogeneous prime ideal defining a nonsingular projective variety such that in(I) is square-free for some monomial order. Then $\mathbb{K}\left[\mathrm{x}_{1}, \ldots, \mathrm{x}_{n}\right] /$ in(I) is Cohen-Macaulay.

2010 Mathematics Subject Classification. 13P10, 13F55, 05E40, 14B07, 13A35 , 13 F50.

Key words and phrases. Gröbner deformations, smoothings, acyclic simplicial complexes, CohenMacaulay, rational singularities. 
Problem 3.6 in [Var19] asks for a counter example of the above conjecture, while Question 4.2 in [CV18] asks if a stronger form of the conjecture is true, namely it asks if the following holds.

Conjecture 2. Let $\mathrm{I} \subset \mathbb{K}\left[\mathrm{x}_{1}, \ldots, \mathrm{x}_{\mathrm{n}}\right]$ be a homogeneous prime ideal defining a nonsingular projective variety such that in(I) is square-free for some monomial order. Then $\mathbb{K}\left[\mathrm{x}_{1}, \ldots, \mathrm{x}_{\mathrm{n}}\right] / \mathrm{in}(\mathrm{I})$ is Cohen-Macaulay with negative a-invariant.

Conjecture 2 is still open for projective curves, in which case it would imply that a nonsingular projective curve with a square-free Gröbner degeneration is forced to have genus zero.

Further motivation for this approach comes from algebras with straightening laws (ASL). These are $\mathbb{K}$-algebras whose generators and relations are governed by a finite poset. All ASL have a discrete counterpart given by a square-free monomial ideal, which can be obtained by a Gröbner degeneration under a degrevlex monomial order. In particular, all ASL fit our setting. In [Eis80] and [DCEP82] De Concini, Eisenbud and Procesi expressed the feeling that a graded ASL R over a field of characteristic zero should have rational singularities as soon as it is a domain. It was quickly realised that this cannot be true in full generality, since there exist graded ASL which are nonnormal domains [HW85]. In unpublished work (mentioned in [DCEP82, p. 11]), Buchweitz proved that in characteristic zero an ASL has rational singularities provided that $R$ has rational singularities on the punctured spectrum and that the discrete part of $R$ (and thus $R$ itself) is Cohen-Macaulay. We are able to infer Buchweitz theorem without the hypothesis that the discrete part of $R$ is Cohen-Macaulay, which we prove is a consequence of $R$ having rational singularities on the punctured spectrum (see Corollary 3.7).

Conjecture 2 trivially holds for hypersurfaces by the following simple, but intriguing, fact. If $f \in \mathbb{K}\left[x_{1}, \ldots, x_{n}\right]$ is a homogeneous polynomial of degree $n$ defining a nonsingular projective hypersurface, then in(f) cannot be a square-free monomial for any monomial order. In our second approach, where we fix a Stanley-Reisner ideal, the above fact translates to "the boundary of a simplex is not Gröbner-smoothable". Smoothings do not always exist, but when they exist, how do they look like? Finding explicit equations, even in seemingly simple cases as $n$-cycles, is a hard problem. We focus our search to Gröbner smoothings, and start by looking at complete intersections. In this case, smoothings always exist and are obtained by unobstructed generic lifting of first order deformations. It turns out that, just as in the case of hypersurfaces, complete intersections using all the variables are never Gröbner-smoothable (Corollary 4.2). It is our general feeling that Gröbner smoothings of Stanley-Reisner schemes are in fact rare. For example, they do not exist for any combinatorial manifold if the order is lexicographic (Proposition 4.12). Another reason for looking at these special deformations is to identify which deformations can be Gröbner. To our knowledge there is no general method to determine if a given abstract deformation is Gröbner. A valuable tool in this approach is provided by Altmann and Christophersen [AC04], who give complete characterisations of the first two cotangent functors in homological terms related to the simplicial complex.

In Proposition 2.4 we prove that the conjectures 1 and 2 are equivalent, by showing that Conjecture 1 in dimension $\leq \mathrm{d}+1$ implies Conjecture 2 in dimension $\leq \mathrm{d}$. For arbitrary monomial orders we know Conjecture 1 for projective curves: this follows by [KS95] if the base field is algebraically closed, and by [Var09] in general (see Proposition 2.6). In Section 3 we show Conjecture 2 when the monomial order is a degree reverse lexicographic one (see Corollary 3.4). In the last section we study Gröbner deformations which are not necessarily associated to degrevlex orders. Conjectures 1 and 2 are equivalent to: A 
simplicial complex which is Gröbner-smoothable over $\mathbb{K}$ must be Cohen-Macaulay and acyclic over $\mathbb{K}$. We prove the following.

1. A combinatorial sphere whose Stanley-Reisner ring is a complete intersection (e.g. the boundary of a cross-polytope) is not Gröbner-smoothable over any field (Corollary 4.2).

2. A graph with exactly one cycle is not Gröbner-smoothable over any real number field (Theorem 4.3).

3. A Gröbner-smoothable graph (over some field) has leafs (Corollary 4.10).

4. Any simplicial complex that is Gröbner-smoothable for the lexicographic order has some free face (Proposition 4.12).

Theorem 4.3 is a statement on nonsingular projective curves of genus one, and its proof relies on a result from number theory by Elkies [Elk87]. The proofs of the other three results are essentially combinatorial.

Acknowledgements: We are grateful to Klaus Altmann and Jan Christophersen for the many useful hints on deformation theory; to Anurag Singh for valuable discussions on many topics touched by this paper, especially on the behaviour of the singularities of Proj $R$ as the graded structure on $R$ varies; to Bhargav Bhatt for clarifications around Theorem 4.3; to Mitra Koley for pointing at the generalisation of the result of Fedder and Watanabe crucial for the proof of the positive characteristic case of Corollary 3.4; to Mateusz Michalek for many experiments on Theorem 4.3.

\section{The CONJECTURES}

Let $S$ be the polynomial ring $\mathbb{K}\left[x_{1}, \ldots, x_{n}\right]$ over a field $\mathbb{K}$ equipped with a positive grading (i.e. $\operatorname{deg} x_{i}>0$ for all $i=1, \ldots, n$ ). For a homogeneous ideal $I \subset S$, let $R=S / I$ and denote by $H_{\mathfrak{m}}^{i}(R)$ the ith local cohomology module of $R$ supported at the unique homogeneous maximal ideal $\mathfrak{m}$ of $R$. The $S$-module $H_{\mathfrak{m}}^{i}(R)$ is $\mathbb{Z}$-graded; for $j \in \mathbb{Z}$, we will denote by $H_{\mathfrak{m}}^{i}(R)_{j}$ its degree $\mathfrak{j}$ part.

We recall that $R$ is Cohen-Macaulay if and only if $H_{\mathfrak{m}}^{i}(R)=0$ for all $i<\operatorname{dim} R$. The a-invariant of a Cohen-Macaulay positively graded $\mathbb{K}$-algebra $R$ is the maximum $j \in \mathbb{Z}$ such that $H_{\mathfrak{m}}^{\operatorname{dim} R}(R)_{j} \neq 0$. In this case, the a-invariant of $R$ is negative if and only if $H^{\operatorname{dim} X}\left(X, \mathcal{O}_{X}\right)=0$ (where $X=$ Proj $R$ ), so having negative a-invariant depends only on $X$. The ring $\mathrm{R}$ is called generalised Cohen-Macaulay if and only if $\mathrm{H}_{\mathfrak{m}}^{i}(\mathrm{R})$ has finite length for any $i<\operatorname{dim} R$. In positive characteristic, $R$ is called $F$-injective if the natural Frobenius action on $H_{\mathfrak{m}}^{i}(R)$ is injective for all $i \in \mathbb{N}$. For the definitions of rational singularities and F-rational singularities we refer to [BH93, Chapter 10].

Remark 2.1. A graded, finitely generated R-module $M$ has finite length if and only if $\operatorname{Supp} M=\{\mathfrak{m}\}$. Using graded duality, it is not difficult to realise that $R$ is generalised Cohen-Macaulay if and only if $R$ is equidimensional and Cohen-Macaulay on the punctured spectrum Spec $R \backslash\{\mathfrak{m}\}$. The latter condition is implied by $R$ being an equidimensional isolated singularity, or, in characteristic zero, by $\mathrm{R}$ being equidimensional and having rational singularities on the punctured spectrum.

Remark 2.2. In the standard graded situation, $R$ is an isolated singularity if and only if $X=\operatorname{Proj} R$ is a nonsingular projective scheme. In the nonstandard graded polynomial ring one has to be careful: For instance, $R$ being an isolated singularity is not equivalent to Proj R being nonsingular (e.g. the weighted projective space $\mathbb{P}(1,1,2)$ is singular).

For convenience, we recall the results of [CV18] which we will most frequently use. 
Theorem 2.3 ([CV18]). Let I $\subset \mathrm{S}$ be a homogeneous ideal such that in(I) is radical for some monomial order. Then

(i) $\operatorname{dim}_{K} H_{\mathfrak{m}}^{\mathrm{i}}(\mathrm{S} / \mathrm{I})_{\mathrm{j}}=\operatorname{dim}_{\mathrm{K}} \mathrm{H}_{\mathfrak{m}}^{\mathrm{i}}(\mathrm{S} / \mathrm{in}(\mathrm{I}))_{j}$ for all $i, j \in \mathbb{Z}$.

(ii) S/I is Cohen-Macaulay if and only if $\mathrm{S} / \mathrm{in}(\mathrm{I})$ is Cohen-Macaulay.

(iii) S/I is generalised Cohen-Macaulay if and only if S/in(I) is Buchsbaum.

(iv) S/I satisfies Serre condition $\left(S_{r}\right)$ if and only if $S /$ in $(I)$ satisfies Serre condition $\left(S_{r}\right)$, for all $\mathrm{r} \geq 1$.

All the above results, even if originally stated for the standard grading, hold true for any positive grading [CV18, Remark 2.5].

For the remaining part of this section, the graded structure on $S=\mathbb{K}\left[x_{1}, \ldots, x_{n}\right]$ will be the standard one.

Proposition 2.4. Conjectures 1 and 2 are equivalent.

Proof. Obviously 2 implies 1 , so let us assume 1 is true and take a homogeneous prime ideal $\mathrm{I} \subset \mathrm{S}=\mathbb{K}\left[\mathrm{x}_{1}, \ldots, x_{n}\right]$ contradicting 2 . We have that $X=$ Proj $S / \mathrm{I}$ is nonsingular, in(I) is square-free for some monomial order, $S / \operatorname{in}(\mathrm{I})$ is Cohen-Macaulay, but the ainvariant of $\mathrm{S} / \mathrm{in}(\mathrm{I})$ is nonnegative. So also $\mathrm{S} / \mathrm{I}$ is Cohen-Macaulay with nonnegative a-invariant. We show now that the Segre embedding $Y=X \times \mathbb{P}^{1} \subset \mathbb{P}^{2 n-1}$ provides a counterexample to Conjecture 1 : Let $A=\mathbb{K}\left[y_{i j}: i \in\{0,1\}, j \in\{1, \ldots, n\}\right]$ and $P \subset A$ be the homogeneous prime ideal defining $Y \subset \mathbb{P}^{2 n-1}$. Then Proj $A / P \cong Y$ is nonsingular and $\mathrm{H}_{M}^{\mathrm{d}}(\mathrm{A} / \mathrm{P})_{0} \cong \mathrm{H}_{\mathfrak{m}}^{\mathrm{d}}(\mathrm{S} / \mathrm{I})_{0} \neq 0$ by [GW78, Theorem 4.1.5], where $\mathrm{M}$ is the unique homogeneous maximal ideal of $A / P$ and $d=\operatorname{dim} S / I$. Since $\operatorname{dim} A / P=d+1$, the ring $A / P$ is not Cohen-Macaulay, so $A / \operatorname{in}(P)$ is not Cohen-Macaulay for any monomial order on $A$. Finally, in $(P)$ is square-free for some special monomial order by [Shi12, Theorem $3.2]$.

Remark 2.5. In the proof of Proposition 2.4 we actually showed that

Conjecture 1 in dimension $\leq \mathrm{d}+1 \Longrightarrow$ Conjecture 2 in dimension $\leq \mathrm{d}$.

Let us recall that there is a one-to-one correspondence between square-free monomial ideals $\mathrm{I}_{\Delta}$ of $\mathrm{S}$ and simplicial complexes $\Delta$ on $[n]=\{1, \ldots, n\}$, given by:

$$
\forall \mathrm{F} \subset[\mathrm{n}], \mathrm{F} \in \Delta \Longleftrightarrow \mathrm{x}:=\prod_{i \in \mathrm{F}} \mathrm{x}_{\mathrm{i}} \notin \mathrm{I}_{\Delta} \text {. }
$$

In some instances it will be convenient to identify $\mathrm{F}$ and $\mathrm{x}_{\mathrm{F}}$, and simply write $\mathbf{x}_{\mathrm{F}} \in \Delta$. An element of $\Delta$ is called face. By maximal face we mean maximal under inclusion. The dimension of a face $F \in \Delta$ is $\operatorname{dim} F=|F|-1$, and the dimension of $\Delta$ is

$$
\operatorname{dim} \Delta=\max \{\operatorname{dim} F: F \in \Delta\} \text {. }
$$

The link of $F \in \Delta$ is the simplicial complex $\operatorname{link}_{\Delta} F=\{G \subset[n]: G \cup F \in \Delta$ and $G \cap F=\emptyset\}$. A simplicial complex $\Delta$ is pure precisely when all its maximal faces have the same dimension. A simplicial complex is strongly connected if and only if for every pair of maximal faces $F, G \in \Delta$, there exist $F=F_{0}, F_{1}, \ldots, F_{r}=G$ maximal faces of $\Delta$ such that $\left|F_{i} \cap F_{i+1}\right|=\left|F_{i}\right|-1$ for all $0 \leq i<r$; in particular, if $\Delta$ is strongly connected then it is pure. We say that $\Delta$ is normal if and only if $\operatorname{link}_{\Delta} F$ is strongly connected for all $F \in \Delta$. Furthermore, $\Delta$ is CohenMacaulay if $\widetilde{H}^{i}\left(\operatorname{link}_{\Delta} \mathrm{F} ; \mathbb{K}\right)=0$ for all $\mathrm{F} \in \Delta$ and $i<\operatorname{dim} \operatorname{link}_{\Delta} \mathrm{F}$, while $\Delta$ is Buchsbaum if $\widetilde{\mathrm{H}}^{\mathrm{i}}\left(\operatorname{link}_{\Delta} \mathrm{F} ; \mathbb{K}\right)=0$ for all $\emptyset \neq \mathrm{F} \in \Delta$ and $i<\operatorname{dim} \operatorname{link}_{\Delta} \mathrm{F}$. These two notions agree with the corresponding algebraic notions for the Stanley-Reisner ring $\mathbb{K}[\Delta]=\mathrm{S} / \mathrm{I}_{\Delta}$. Finally, if $\mathbb{K}[\Delta]$ is Cohen-Macaulay, then it has negative a-invariant if and only if $\widetilde{H}^{\operatorname{dim} \Delta}(\Delta ; \mathbb{K})=0$.

Proposition 2.6. Let $\mathrm{I} \subset \mathrm{S}$ be an ideal such that in $(\mathrm{I})=\mathrm{I}_{\Delta}$ for some monomial order. 
(i) If $\mathrm{S} / \mathrm{I}$ is a domain, then $\Delta$ is strongly connected.

(ii) If $\mathrm{S} / \mathrm{I}$ is a normal domain, then $\Delta$ is normal.

(iii) If $\mathrm{I}$ is homogeneous and $\operatorname{Proj} \mathrm{S} / \mathrm{I}$ is an equidimensional nonsingular projective scheme, then $\Delta$ is Buchsbaum.

Proof. Point (i) follows by [KS95] if the base field is algebraically closed, and by [Var09] in general.

(ii) Since $R / I$ is normal, it satisfies Serre condition $\left(S_{2}\right)$, so, by Theorem 2.3 (iv), $\mathbb{K}[\Delta]$ satisfies $\left(S_{2}\right)$ as well; on the other hand, it is known and easy to prove that $\Delta$ is normal if and only if $\mathbb{K}[\Delta]$ satisfies $\left(S_{2}\right)$.

(iii) As $\mathrm{S} / \mathrm{I}$ is generalised Cohen-Macaulay, we conclude by Theorem 2.3 (iii).

Given a simplicial complex $\Delta$ on $\mathfrak{n}$ vertices, we say it is Gröbner-smoothable over $\mathbb{K}$ if there exists a homogeneous prime ideal $\mathrm{I} \subset \mathbb{K}\left[x_{1}, \ldots, x_{n}\right]$ defining a nonsingular projective variety and a monomial order such that in $(\mathrm{I})=\mathrm{I}_{\Delta}$. So a further equivalent formulation of Conjectures 1 and 2, using Proposition 2.4, is the following:

Conjecture 3. A simplicial complex which is Gröbner-smoothable over $\mathbb{K}$ is CohenMacaulay and acyclic over $\mathbb{K}$.

A simplicial complex $\Delta$ is acyclic over $\mathbb{K}$ if all its reduced cohomology with coefficients in $\mathbb{K}$ vanishes.

\section{DeGREe REVERSE LEXICOGRAPHIC DEGENERATIONS}

Throughout this section $S=\mathbb{K}\left[x_{1}, \ldots, x_{n}\right]$ is again allowed to have any positive grading.

Definition 3.1. A monomial order on $S$ is $x_{n}$-addicted if, whenever $f \in S$ and $x_{n} \mid \operatorname{in}(f)$, we have that $x_{n} \mid f$.

Remark 3.2. Any degrevlex monomial order is $x_{n}$-addicted if $x_{n}$ is the smallest variable.

Theorem 3.3. Let $\mathrm{I} \subset \mathrm{S}$ be a homogeneous ideal such that $\mathrm{S} / \mathrm{I}$ is generalised CohenMacaulay and $\mathrm{x}_{\mathrm{n}}$ is $\mathrm{S} / \mathrm{I}$-regular. If in(I) is radical for some $\mathrm{x}_{\mathrm{n}}$-addicted monomial order, then $\mathrm{S} /$ in(I) is Cohen-Macaulay with negative a-invariant.

Proof. By Theorem 2.3 (iii), S/in(I) is generalised Cohen-Macaulay. By Hochster's formula for local cohomology [BH93, Theorem 3.5.8] we infer:

$$
\mathrm{H}_{\mathfrak{m}}^{\mathrm{i}}(\mathrm{S} / \mathrm{in}(\mathrm{I}))=\mathrm{H}_{\mathfrak{m}}^{\mathrm{i}}(\mathrm{S} / \mathrm{in}(\mathrm{I}))_{0} \cong \widetilde{\mathrm{H}}^{\mathrm{i}-1}(\Delta) \quad \forall i \geq 1,
$$

where $\Delta$ is the simplicial complex on $n$ vertices such that $\operatorname{in}(\mathrm{I})=\mathrm{I}_{\Delta}$, the Stanley-Reisner ideal of $\Delta$. Since $\mathrm{H}_{\mathrm{m}}^{0}(\mathrm{~S} / \mathrm{in}(\mathrm{I}))$ trivially vanishes, it is enough to show that $\widetilde{\mathrm{H}^{\mathrm{i}}}(\Delta)=0$ for all $i \in \mathbb{N}$. We will show an even stronger statement, namely, that $\Delta$ is contractible.

In order to do so, it is enough to show that the vertex corresponding to $x_{n}$ is a cone point for $\Delta$, i.e. that $x_{n}$ is contained in every maximal face of $\Delta$. This is equivalent to $x_{n}$ not dividing any minimal monomial generator of in(I). By contradiction, let $u$ be a minimal monomial generator of in(I) which is divisible by $x_{n}$, and let $f \in I$ such that $\operatorname{in}(f)=u$. As the monomial order is $x_{n}$-addicted, there exists $g \in S$ such that $f=x_{n} g$. Since $x_{n}$ is $S / I$-regular, we have $g \in I$. In particular, in $(g)$ is a monomial of in(I) strictly dividing $\mathfrak{u}$, thus contradicting the minimality of $\boldsymbol{u}$.

The following corollary solves Conjectures 1 and 2 for any degree reverse lexicographic monomial order.

Corollary 3.4. Let I $\subset \mathrm{S}$ be homogeneous prime ideal such that in(I) is a square-free monomial ideal with respect to a degrevlex monomial order. 
(i) If S/I is generalised Cohen-Macaulay, then S/in(I) (and thus also S/I) is CohenMacaulay with negative a-invariant.

(ii) In characteristic zero, S/I has rational singularities whenever it has rational singularities on the punctured spectrum.

(iii) In positive characteristic, S/I is F-rational whenever it is F-rational on the punctured spectrum.

Proof. (i) We can assume that $x_{1}>\ldots>x_{n}$. If $x_{n} \notin \mathrm{I}$, then we conclude by Theorem 3.3. Otherwise, set $S^{\prime}=S /\left(x_{n}\right)$ and $I^{\prime}=I /\left(x_{n}\right)$. Then $I^{\prime} \subset S^{\prime}$ is a prime ideal such that $S^{\prime} / I^{\prime} \cong S / I$ is generalised Cohen-Macaulay and $\operatorname{in}\left(I^{\prime}\right)=\operatorname{in}(I) /\left(x_{n}\right)$ is a squarefree monomial ideal with respect to the degrevlex monomial order extending the linear order $x_{1}>\ldots>x_{n-1}$. By induction on the number of variables, $S^{\prime} / \operatorname{in}\left(I^{\prime}\right) \cong S /$ in $(I)$ is Cohen-Macaulay with negative a-invariant.

(ii) It follows from (i) and [Wat83, Theorem 2.2].

(iii) Since in(I) is a square-free monomial ideal, $\mathrm{S} /$ in(I) is F-injective. By (i), S/ in(I) is Cohen-Macaulay, so we get that S/I is F-injective using [CH97, Theorem 2.1]. So, using (i), we have that $\mathrm{R}$ is Cohen-Macaulay, F-injective, F-rational on the punctured spectrum, and has negative a-invariant. In [FW89, Theorem 2.8, Remark 1.17], Fedder and Watanabe proved that these conditions, under the stronger assumption that $R$ is an isolated singularity, imply that $\mathrm{R}$ is F-rational. It turns out that their proof works as well without the stronger assumption (see [Kol18, Theorem 5.49, Lemma 5.44] for a rigorous proof), hence we conclude.

Remark 3.5. Corollary 3.4 solves in positive Conjectures 1 and 2 for a degree reverse lexicographic monomial order even. In fact, it uses only the weaker assumption that Proj S/I is Cohen-Macaulay, instead of nonsingular. However, this stronger version of Conjectures 1 and 2 is not true for other monomial orders, as the following examples show.

Example 3.6. (i) Let $\mathrm{f}=x \mathrm{yz}+\mathrm{y}^{3}+z^{3} \in \mathrm{S}=\mathbb{K}[x, y, z]$, I $=(\mathrm{f})$ and choose the lexicographic monomial order with $\mathrm{x}>\mathrm{y}>z$. Then $\mathrm{I}$ is a homogeneous (w.r.t. the standard grading) prime ideal, Proj S/I is Cohen-Macaulay and in(I) $=(x y z)$ is square-free. However S/in(I) is Cohen-Macaulay with a-invariant 0, so Conjecture 2 is false if we replace "Proj S/I is nonsingular" by "Proj S/I is Cohen-Macaulay".

(ii) In [Var19, Example 3.4], a homogeneous prime ideal I of the standard graded polynomial ring $S$ in 6 variables over $\mathbb{K}$ is considered. That ideal satisfies: Proj $\mathrm{S} / \mathrm{I}$ is a Cohen-Macaulay surface, in(I) is square-free for a lexicographic monomial order, but $\mathrm{S} /$ in(I) is not Cohen-Macaulay. So also Conjecture 1 is false if we replace "Proj S/I is nonsingular" by "Proj S/I is Cohen-Macaulay".

In the following corollary, the notion "graded algebra with straightening laws" is the same used in [BV88].

Corollary 3.7. Let $\mathrm{R}$ be a graded algebra with straightening laws over a field such that $\mathrm{R}$ is a domain.

(i) If $\mathrm{R}$ is generalised Cohen-Macaulay, then $\mathrm{R}$ is Cohen-Macaulay with negative ainvariant.

(ii) In characteristic zero, $\mathrm{R}$ has rational singularities whenever it has rational singularities on the punctured spectrum.

(iii) In positive characteristic, $\mathrm{R}$ is $\mathrm{F}$-rational whenever it is F-rational on the punctured spectrum.

Proof. Let $\Omega$ be the set of ASL generators of R, i.e. the partially ordered $\mathbb{K}$-algebra generators of $R$ to which the straightening laws apply. This means that $R \cong S / I$ where 
$S=\mathbb{K}\left[x_{\omega}: \omega \in \Omega\right]$ is the polynomial ring over $\mathbb{K}$ whose variables correspond to the elements of $\Omega$ and I $\subset S$ is a prime ideal such that in(I) is a square-free monomial ideal with respect to any degrevlex monomial order on $S$ extending the partial order of $\Omega$. By supplying $S$ with the degrees given by $\operatorname{deg}\left(x_{\omega}\right)=\operatorname{deg}(\omega)$ for all $\omega \in \Omega$, I is a homogeneous ideal of $\mathrm{S}$, so we conclude by Corollary 3.4.

Remark 3.8. If $R=S / I$ is an isolated singularity, the hypothesis of all three parts of Corollary 3.4 and Corollary 3.7 are fulfilled.

\section{GrÖBner DEFormations FOR ARBitraRY MONOMIAL ORDERS}

From the remaining part of this article we will assume that the graded structure on $S=\mathbb{K}\left[x_{1}, \ldots, x_{n}\right]$ is the standard one.

4.1. Complete intersections. Complete intersections are a particularly nice case to consider: they are always smoothable (in fact there are no obstructions to lifting infinitesimal deformations), however we are going to see that square-free monomial complete intersections using all the variables of $\mathbb{K}\left[x_{1}, \ldots, x_{n}\right]$ in their minimal generators are not Gröbner-smoothable. The proof of this result is a simple combinatorial argument, that however we find instructive: it is a generalisation of the easy fact that a polynomial defining a Calabi-Yau hypersurface cannot have a square-free initial monomial, which was one of the starting points of this paper.

Let $I=\left(u_{1}, \ldots, u_{c}\right)$ be a monomial ideal of $S=\mathbb{K}\left[x_{1}, \ldots, x_{n}\right]$ minimally generated by monomials $u_{1}, \ldots, u_{c}$ of degree $d_{i}=\operatorname{deg} u_{i}$. Then $I$ is a complete intersection (of height c) if and only if the $u_{i}$ 's are supported on disjoint sets of variables. So, if $I=I_{\Delta}$ is squarefree, then $d_{1}+\ldots+d_{c} \leq n$ with equality holding if and only if $\mathbb{K}[\Delta]$ has a-invariant 0 .

Let $\mathrm{I}=\mathrm{I}_{\Delta} \subset \mathfrak{m}^{2}$ be a complete intersection such that $\mathbb{K}[\Delta]$ has a-invariant 0 . Then $\Delta$ is the join of $\mathrm{c}$ boundaries of simplices. So let $\Delta=\partial \Delta_{1} * \cdots * \partial \Delta_{\mathrm{c}}$, with $\operatorname{dim} \Delta_{\mathrm{i}}=\mathrm{d}_{\mathrm{i}}-1 \geq 1$. Let the variables corresponding to $\Delta_{i}$ be $x_{i 1}, \ldots, x_{i d_{i}}$, so we have

$$
\mathrm{I}_{\Delta}=\left(\mathrm{x}_{11} \ldots \mathrm{x}_{1 \mathrm{~d}_{1}}, \ldots, \mathrm{x}_{\mathrm{c} 1} \ldots \mathrm{x}_{\mathrm{cd}}\right) \text {. }
$$

Let $\tau$ be some monomial order, and assume that $I=\left(f_{1}, \ldots, f_{c}\right)$ is a reduced homogeneous Gröbner basis with

$$
f_{i}=x_{i 1} \ldots x_{i d_{i}}-\sum \lambda_{M} M, \text { where } \lambda_{M} \in \mathbb{K} \text { and } M \leq_{\tau} x_{i 1} \ldots x_{i d_{i}} .
$$

As the Gröbner basis is homogeneous and reduced, all the monomials $M$ above have degree $d_{i}$ and are supported on faces of $\Delta$. Without losing generality we may always assume that we have the following inequalities for $\tau$ :

$$
\begin{aligned}
x_{i 1}>\ldots & >x_{i d_{i}} \\
x_{11} \geq x_{i j} & \forall x_{i j}, \\
x_{i j} \geq x_{c d_{c}} & \forall x_{i j} \text { with } i>1 .
\end{aligned}
$$

Lemma 4.1. With the above convention, if $\left\{\mathrm{f}_{1}, \ldots, \mathbf{f}_{\mathrm{c}}\right\}$ is a reduced Gröbner basis of the ideal I it generates, then the point $[1: 0: \cdots: 0]$ is a singular point of Proj S/I, where the first projective coordinate corresponds to the variable $\mathrm{x}_{11}$.

Proof. First of all, as $x_{11}^{a}$ is the largest monomial of degree $a$, it does not appear in the support of any $f_{i}$, so $[1: 0: \cdots: 0] \in \operatorname{Proj} S /$ I. Assume the contrary, namely that $[1: 0: \cdots: 0]$ is a smooth point of Proj $S / I$. Then the Jacobian computed at $[1: 0: \cdots: 0]$ has to have maximal rank $c$. This implies that no row can be zero when substituting. In particular, in the support of $f_{c}$, we must have some monomial of the form $x_{11}^{d_{c}-1} x_{i j}$. If $i>1$, then $x_{c d_{c}}<x_{i j}$. Because all other variables $x_{c k}<x_{11}$, we have $x_{c 1} \ldots x_{c d_{c}}<x_{11}^{d_{c}-1} x_{i j}$, 
contradicting in $\left(f_{c}\right)=x_{c 1} \ldots x_{c d_{c}}$. So the monomial has to be $x_{11}^{d_{c}-1} x_{1 j}$ for some $j$. This implies two things:

$$
\begin{array}{ll}
x_{11} x_{1 j} \in \Delta & \text { because the Gröbner basis is reduced. } \\
x_{1 j}<x_{c_{c}} & \text { because } x_{c 1} \ldots x_{c_{c}}>x_{11}^{d_{c}-1} x_{1 j} .
\end{array}
$$

From (2) we obtain that $d_{1}>2$. Given that the row of the Jacobian corresponding to $f_{1}$ cannot be zero when substituting $[1: 0: \cdots: 0]$, we must have some monomial $x_{11}^{\mathrm{d}_{1}-1} x_{\mathrm{kl}}$ in the support of $f_{1}$. We claim that this leads to a contradiction. There are two cases: If $k=1$, then $x_{11}^{d_{1}-1} x_{1 l}>x_{11} \ldots x_{1 d_{1}}$ which is a contradiction to in $\left(f_{1}\right)=x_{11} \ldots x_{1 d_{1}}$. If $k>1$, then by (3) we have $x_{1 j}<x_{c d_{c}}$, and by assumption (1) we have $x_{c d_{c}}<x_{k l}$. So $x_{11} \ldots x_{1 d_{1}}<x_{11}^{d_{1}-1} x_{1 j}<x_{11}^{d_{1}-1} x_{k l}$ which is again a contradiction to $\operatorname{in}\left(f_{1}\right)=x_{11} \ldots x_{1 d_{1}}$.

Corollary 4.2. A join of boundaries of positive dimensional simplices is not Gröbnersmoothable over any field $\mathbb{K}$.

The simplicial complexes of the above corollary are particular combinatorial spheres. If Conjecture 3 is true, no combinatorial sphere is Gröbner-smoothable. As a consequence of the results in the next subsection, this is at least true for 1-dimensional spheres.

4.2. Dimension 1. In Proposition 2.4 we proved that if Conjecture 1 is true in dimension $\leq \mathrm{d}+1$, then Conjecture 2 is true in dimension $\leq \mathrm{d}$. On the other hand Conjecture 1 is true whenever $\operatorname{dim} S / \mathrm{I} \leq 2$ by Proposition 2.6 (since a 1-dimensional simplicial complex is Cohen-Macaulay if and only if it is strongly connected), so the first instance where it is open is when $\operatorname{dim} S / I=3$. This justifies our next interest, that is to study Conjecture 2 when $\operatorname{dim} S / I=2$. In this case, Conjecture 2 says that if $\mathrm{I} \subset \mathrm{S}$ defines a smooth projective curve $\mathrm{C}$ and in(I) is square-free for some monomial order, then the genus of $\mathrm{C}$ must be zero. While the most general case remains open, in this section we provide some evidence for it.

4.2.1. Elliptic curves over real number fields. In the proof of the next theorem we use the result of Elkies [Elk87] which states that an elliptic curve over the rational numbers has infinitely many supersingular primes.

Theorem 4.3. If $\mathrm{I} \subset \mathbb{Q}\left[\mathrm{x}_{1}, \ldots, \mathrm{x}_{n}\right]$ defines a nonsingular projective curve of genus one, then in(I) is not square-free for any monomial order.

Proof. Given a prime number $p$, we will denote by $S_{p}$ the polynomial $\operatorname{ring} \mathbb{Z} / p \mathbb{Z}\left[x_{1}, \ldots, x_{n}\right]$. Also, for an ideal $J \subset S=\mathbb{Q}\left[x_{1}, \ldots, x_{n}\right]$ we write $J_{p}$ for the ideal $J^{\prime} S_{p} \subset S_{p}$, where $J^{\prime}=J \cap \mathbb{Z}\left[x_{1}, \ldots, x_{n}\right]$.

Let $E \subset \mathbb{P}^{n-1}$ be the nonsingular projective curve of genus 1 defined by $\mathrm{I} \subset \mathrm{S}$. Of course $I_{p}$ defines $E_{p}$, its reduction mod $p$, for all prime numbers $p$. Since any nonsingular projective curve of genus one can be embedded as an elliptic curve in $\mathbb{P}^{2}$, there exist infinitely many supersingular primes $p$ for $E$ by [Elk87, Theorem 1]. This means that the Frobenius does not act injectively on $H^{1}\left(E_{p}, \mathcal{O}_{E_{p}}\right)$ for infinitely many primes $p$. Since $H^{1}\left(E_{p}, \mathcal{O}_{E_{p}}\right) \cong H_{\mathfrak{m}_{p}}^{2}\left(S_{p} / I_{p}\right)_{0}$, this implies

$$
S_{p} / I_{p} \text { is not } F \text {-injective for infinitely many primes } p \text {. }
$$

Suppose by contradiction that in(I) is square-free for some monomial order. So in(I) = $\mathrm{I}_{\Delta}$ for some 1-dimensional simplicial complex $\Delta$. By Proposition 2.6 (i) $\Delta$ must be a connected 1-dimensional simplicial complex, thus $\Delta$ is shellable, and thus $\mathrm{S} / \mathrm{I}_{\Delta}$ is CohenMacaulay. Looking at the Buchberger algorithm, it is easy to realise that in $\left(I_{p}\right)=\left(I_{\Delta}\right)_{p}$ for all prime numbers $p \gg 0$. So $S_{p} / \operatorname{in}\left(I_{p}\right)$ is F-injective and Cohen-Macaulay for all 
$p \gg 0$; therefore $S_{p} / I_{p}$ is F-injective by [CH97, Theorem 2.1] for all $p \gg 0$ as well, a contradiction to (4).

Remark 4.4. With the appropriate notion of reduction mod $p$, the proof of Theorem 4.3 works replacing $\mathbb{Q}$ with any number field $\mathbb{K} \subset \mathbb{R}$ by [Elk89]. The proof fails however on a general field of characteristic zero. For example, all the reductions mod $p$ of the ring $\mathbb{Q}(\pi)[x, y, z] /\left(x^{3}+y^{3}+z^{3}+\pi \cdot x y z\right)$ are F-injective for all prime numbers $p$, thus (4) fails.

4.2.2. Leafless 1-dimensional complexes. Theorem 4.3 together with Remark 4.4 say that a graph with one cycle is not Gröbner-smoothable over a real number field. We extend this to arbitrary fields and any number of cycles, with the extra assumption that the simplicial complex involved has no leafs. By a leaf we mean a vertex which is contained in exactly one maximal face. We call a simplicial complex leafless if it has no leafs.

Remark 4.5. Recall that a free face of a pure d-dimensional simplicial complex is a face of dimension $\mathrm{d}-1$ contained in only one maximal face. The notion of pure simplicial complex with no free faces coincides with the notion of leafless pure simplicial complex only in dimension 1. In higher dimension, leafless is implied by the absence of free faces.

We start with the setup. Since $I \subset S=\mathbb{K}\left[x_{1}, \ldots, x_{n}\right]$ is a homogeneous prime ideal such that $\operatorname{dim} S / I=2$ and $\operatorname{in}(\mathrm{I})=\mathrm{I}_{\Delta}$ for some monomial order, then by Proposition 2.6 one has that $\Delta$ is a 1 -dimensional, connected simplicial complex on $[n]$, i.e., $\Delta$ is a connected graph on $n$ vertices. Moreover, from now on we will assume that $\mathrm{I}$ does not contain linear forms (harmless for our goals), that is, $\{i\} \in \Delta$ for every $i \in[n]$. Let $\left\{f_{\mathrm{F}} \mid \mathrm{F}\right.$ is a minimal non-face of $\left.\Delta\right\}$ be a homogeneous reduced Gröbner basis of $\mathrm{I}_{\Delta}$ with respect to some monomial order $\tau$, such that

$$
\operatorname{in}_{\tau} f_{F}=x_{F} \text { for every minimal } F \notin \Delta \text {. }
$$

We also use the notation

$$
\mathrm{d}_{\mathrm{F}}:=\operatorname{deg} \mathrm{f}_{\mathrm{F}} \text { for every minimal } \mathrm{F} \notin \Delta .
$$

As $\Delta$ is 1-dimensional, $d_{\mathrm{F}}$ is either two or three. We may and will relabel the vertices of $\Delta$ in such a way that $\operatorname{link}_{\Delta} 1=\{2, \ldots, r\}$ (we abuse here notation and write just 2 for the face $\{2\}$ and so on). For the fixed monomial order $\tau$ we may always assume without loss of generality that we have the following inequalities:

$$
\begin{aligned}
& x_{1}>x_{i} \quad \forall i>1, \\
& x_{2}>\cdots>x_{r} .
\end{aligned}
$$

Remark 4.6. If $\{1, l\} \notin \Delta$, then $x_{1}^{d_{F}-1} x_{l} \notin \operatorname{supp} f_{F}$ and $x_{1}^{d_{F}} \notin \operatorname{supp} f_{F}$ for every minimal non-face $F$.

Proof. This follows directly from the fact that $x_{1}$ is the highest variable, and the Gröbner basis is reduced.

Lemma 4.7. For $1<\mathrm{a} \leq \min \{\mathrm{r}, 3\}$, we have that

$$
x_{1}^{d_{\mathrm{F}}-1} x_{\mathrm{a}} \notin \operatorname{supp} \mathrm{f}_{\mathrm{F}} \text {, for all minimal non-faces with } 1 \notin \mathrm{F} \text {. }
$$

Proof. We label the minimal non-faces of $\Delta$ not containing 1 by $F_{1}, \ldots, F_{s}$, denote the corresponding degrees by $d_{1}, \ldots, d_{s}$, and assume that

$$
\text { if } 1 \leq i<j \leq s \text {, then } x_{1}^{3-d_{i}} \operatorname{in}_{\tau} f_{F_{i}}<x_{1}^{3-d_{j}} \operatorname{in}_{\tau} f_{F_{j}} \text {. }
$$

We will prove (7) inductively, that is, we assume that $(7)$ holds for $F_{1}, \ldots, F_{k-1}$ and prove that it also holds for $F_{k}$. 
Let $a=2$ and assume that $x_{1}^{d_{k}-1} x_{2} \in \operatorname{supp} f_{F_{k}}$. This implies that $x_{1}^{d_{k}-1} x_{2}<x_{F}$, so that $x_{2}<x_{i}$ for all $i \in F_{k}$. Since $x_{2}<x_{i}$ implies $i>r$ by (6), it follows that $\{1, i\} \notin \Delta$ for every $i \in F_{k}$. Let $i_{k}$ be one of the vertices in $F_{k}$ and build the S-polynomial

$$
S\left(f_{\left\{1, i_{k}\right\}}, f_{F_{k}}\right)=x_{F_{k} \backslash i_{k}} f_{\left\{1, i_{k}\right\}}-x_{1} f_{F_{k}} \text {. }
$$

As we are dealing with a Gröbner basis, we must have

$$
S\left(f_{\left\{1, i_{k}\right\}}, f_{F_{k}}\right)=\sum g_{F} \cdot f_{F} \text {, with in }\left(g_{F} \cdot f_{F}\right)<x_{1} x_{F_{k}} .
$$

The highest exponent of $x_{1}$ in a monomial in the support of $\mathbf{x}_{\mathrm{F}_{k} \backslash i_{k}} f_{\left\{1, i_{k}\right\}}$ is 1 . So, because $x_{1}^{d_{k}} x_{2} \in \operatorname{supp} x_{1} f_{F_{k}}$ with $d_{k}>1$, we have $x_{1}^{d_{k}} x_{2} \in \operatorname{supp} S\left(f_{\left\{1, i_{k}\right\}}, f_{F_{k}}\right)$. Thus, there must appear some non-face $F^{\prime}$ on the right hand side of (9), such that

$$
x_{1}^{d_{k}} x_{2} \in \operatorname{supp} g_{F^{\prime}} f_{F^{\prime}} \text {. }
$$

Claim 1. For every $\mathrm{F}^{\prime}$ as above we have $1 \notin \mathrm{F}^{\prime}$.

Proof of Claim 1: We assume that $1 \in \mathrm{F}^{\prime}$ and seek a contradiction. There are two cases: If $\left|F^{\prime}\right|=3$, then let $F^{\prime}=\{1, l, m\}$, so $l, m \in \operatorname{link}_{\Delta} 1$. In particular $x_{l} \leq x_{2}$ and $x_{m} \leq x_{2}$, so $x_{1}^{2} x_{2}>x_{1} x_{l} x_{m}=\operatorname{in}\left(f_{F^{\prime}}\right)$. This implies $\operatorname{in}\left(g_{F^{\prime}} f_{F^{\prime}}\right)<x_{1}^{d_{k}} x_{2}$, so $x_{1}^{d_{k}} x_{2} \notin \operatorname{supp} g_{F^{\prime}} f_{F^{\prime}}$, which is a contradiction to (10).

If $\left|F^{\prime}\right|=2$, then let $F^{\prime}=\{1, l\}$. Because $x_{1}^{2} \notin \operatorname{supp} f_{F^{\prime}}$ we must have by (10) that $x_{1}^{d_{k}-1} \in \operatorname{supp} g_{F^{\prime}}$, thus also that $x_{1}^{d_{k}} x_{l} \in \operatorname{supp} g_{F^{\prime}} f_{F^{\prime}}$. By Remark 4.6, we have that $x_{1}^{d_{k}} x_{l} \notin \operatorname{supp} S\left(f_{\left\{1, i_{k}\right\}}, f_{F_{k}}\right)$, so $x_{1}^{d_{k}} x_{l}$ must cancel out with some monomial in $\operatorname{supp}\left(g_{F} f_{F}\right)$ for some F. But, again by Remark 4.6, we have $x_{1}^{d_{k}} x_{l} \notin \operatorname{supp}\left(g_{F} f_{F}\right)$ for every F and we also obtain a contradiction.

By Claim 1 we have $F^{\prime}=F_{i}$ for some $i \in\{1, \ldots, s\}$. As $x_{1}^{d_{F^{\prime}}} \notin \operatorname{supp} f_{F^{\prime}}$, we must have $x_{1}^{d_{F^{\prime}-1}} x_{2} \in \operatorname{supp} f_{F^{\prime}}$. By the inductive hypothesis we must have that $i \geq k$. From (8) it follows that $x_{1}^{3-d_{0}}$ in $f_{F^{\prime}} \geq x_{1}^{3-d_{k}}$ in $f_{F_{k}}$. Thus we get $\operatorname{in}_{\tau} g_{F^{\prime}} f_{F^{\prime}} \geq x_{1} x_{F_{k}}$, which is a contradiction to (9).

When $a=3$, the only change in the proof is in the first lines: From $x_{3}<x_{i}$ for $i \in F_{k}$ it follows that there exists $j \in F_{k}$ with $\{1, j\} \notin \Delta$ (for $x_{2}$ we had this for any $i \in F_{k}$ ). The rest of the proof runs analogously.

The proof of Lemma 4.7 no longer works for the third-largest variable in the link of 1 , namely $x_{1} x_{4} \in \operatorname{supp}_{\{2,3\}}$ would not lead to a similar contradiction. A straight-forward generalisation of the argument in higher dimension is also not possible, because the link of 1 is no longer a 0 -dimensional complex, so we may no longer assume that $x_{2}>\cdots>x_{r}$. So Lemma 4.7 is the best possible result using this type of arguments related to monomial orders and reduced Gröbner bases.

Remark 4.8. For a minimal non-face $F$ with $|F|=3$ and $1 \in F$, we have

$$
x_{1}^{2} x_{2}, x_{1}^{2} x_{3} \notin \operatorname{supp} f_{F} .
$$

Proof. Because $F=\{1, l, m\}$ is a minimal non-face, we have $l, m \in \operatorname{link}_{\Delta} 1$. As we chose $x_{2}$ and $x_{3}$ to be the largest two variables in the link of 1 , we have $x_{2} \geq x_{1}$ and $x_{3} \geq x_{m}$ (or $l$ and $m$ switched). Thus $x_{1}^{2} x_{2}>x_{1}^{2} x_{3}>x_{1} x_{k} x_{l}$.

Theorem 4.9. Let $\Delta$ be a 1-dimensional simplicial complex, and fix a monomial order. Let $\mathrm{I} \subset \mathrm{S}$ be homogeneous ideal with in $\mathrm{I}=\mathrm{I}_{\Delta}$, and let $\left\{\mathrm{f}_{\mathrm{F}} \mid \mathrm{F}\right.$ is a minimal non-face of $\left.\Delta\right\}$ be a reduced homogeneous Gröbner basis of $\mathrm{I}$. If 1 is not a leaf, then $\mathrm{P}_{1}=[1: 0: \cdots: 0]$ is a singular point of Proj S/I. 
Proof. Clearly $x_{1}^{d_{F}}$ is not in the support of any $f_{F}$, which implies that $P_{1} \in \operatorname{Proj} S / I$. Because 1 is not a leaf we have $r \geq 3$, where $\{2, \ldots, r\}=\operatorname{link}_{\Delta} 1$. The Jacobian Jac(I) can be split in two blocks with full rows as follows:

$B_{1}:=$ the $n-r$ rows corresponding to the degree 2 generators $f_{F}$ with $x_{1} \mid x_{F}$.

$B_{2}:=$ the rows corresponding to the $f_{F}$ without $x_{1}^{d_{F}-1} x_{2}$ and $x_{1}^{d_{F}-1} x_{3}$ in their support.

When substituting $P_{1}$ in the Jacobian, the columns indexed by $x_{r+1}, \ldots, x_{n}$ produce an identity submatrix in $\mathrm{B}_{1}\left(\mathrm{P}_{1}\right)$, so

$$
\operatorname{rank} B_{1}\left(P_{1}\right)=n-r
$$

In $B_{2}\left(P_{1}\right)$, the columns indexed by $x_{r+1}, \ldots, x_{n}$ are all zero because the Gröbner basis is reduced. The first column is zero because $x_{1}^{d_{F}} \notin \operatorname{supp} f_{F}$ for any minimal non-face $F$. By Lemma 4.7 and Remark 4.8 the columns indexed by $x_{2}$ and $x_{3}$ are also zero in $B_{2}\left(P_{1}\right)$. This leaves at most $r-3$ nonzero columns in $B_{2}\left(P_{1}\right)$, thus

$$
\operatorname{rank} B_{2}\left(P_{1}\right) \leq r-3 \text {. }
$$

This means that $\operatorname{rank} \operatorname{Jac}\left(\mathrm{P}_{1}\right) \leq n-r+r-3=n-3<\operatorname{codim}_{\mathbb{P}^{n-1}} \operatorname{Proj} S / I$, so $P_{1}$ is a singular point.

Corollary 4.10. A graph which is Gröbner-smoothable over some field must have leafs. In particular, a cycle is not Gröbner-smoothable over any field.

Remark 4.11. For lexicographic monomial orders the above arguments are much easier and work in any dimension: If $\tau$ is the lexicographic order corresponding to $x_{1}>\cdots>x_{n}$, we have that if $x_{1} \mid M$ with $M \in \operatorname{supp} f_{F}$, then $x_{1} \mid \mathbf{x}_{F}$. Furthermore, $x_{1}^{2}$ does not divide any monomial in the support of any $f_{F}$. So $\left(\frac{\partial f_{F}}{\partial x_{i}}\left(P_{1}\right)\right)_{i=1, \ldots, n} \neq(0, \ldots, 0)$ if and only if $F=\{1, j\}$ where $\{1, j\} \notin \Delta$. This means $P_{1}=[1: 0, \ldots, 0]$ can be a smooth point of $\operatorname{Proj}(S / I)$ only if $\left|\operatorname{link}_{\Delta} 1\right| \leq \operatorname{dim} \Delta$. This leads to the next result.

Proposition 4.12. In any dimension, a leafless simplicial complex $\Delta$ is not Gröbnersmoothable with respect to any lexicographic order. In particular, pseudo-manifolds are not lexicographically Gröbner-smoothable.

Proof. Since $\mathrm{I}_{\Delta}$ is the initial ideal of a prime ideal, it must be strongly connected by Proposition 2.6 (i), and thus pure. So Remark 4.11 yields the result.

\section{REFERENCES}

[AC04] Klaus Altmann and Jan Arthur Christophersen, Cotangent cohomology of Stanley-Reisner rings, Manuscripta Math. 115 (2004), no. 3, 361-378. MR 2102057 (2005j:13020)

[AC10] _ Deforming Stanley-Reisner schemes, Mathematische Annalen 348 (2010), no. 3, 513537.

[BGT97] Winfried Bruns, Joseph Gubeladze, and Ngô Viêt Trung, Normal polytopes, triangulations, and Koszul algebras, Journal fur die reine und angewandte Mathematik 485 (1997), 123-160.

[BH93] Winfried Bruns and Jürgen Herzog, Cohen-Macaulay rings, Cambridge Studies in Advanced Mathematics, vol. 39, Cambridge University Press, Cambridge, 1993. MR 1251956 (95h:13020)

[BR07] Winfried Bruns and Tim Römer, h-vectors of Gorenstein polytopes, Journal of Combinatorial Theory, Series A 114 (2007), no. 1, 65-76.

[BV88] Winfried Bruns and Udo Vetter, Determinantal rings, vol. 1327, Springer-Verlag, Berlin, 1988.

[CH97] Aldo Conca and Jürgen Herzog, Ladder determinantal rings have rational singularities, Advances in Mathematics 132 (1997), no. 1, 120-147.

[Chr11] Jan Arthur Christophersen, Deformations of equivelar Stanley-Reisner abelian surfaces, Advances in Mathematics 227 (2011), no. 2, 801-829.

[CI14] Jan Arthur Christophersen and Nathan Owen Ilten, Degenerations to unobstructed Fano Stanley-Reisner schemes, Math. Z. 278 (2014), no. 1-2, 131-148. MR 3267573 
[CV18] Aldo Conca and Matteo Varbaro, Square-free Gröbner degenerations, arXiv preprint arXiv:1805.11923 (2018).

[DCEP82] Corrado De Concini, David Eisenbud, and Claudio Procesi, Hodge algebras, Astérisque, vol. 91, Société Mathématique de France, Paris, 1982, With a French summary. MR 680936

[Eis80] David Eisenbud, Introduction to algebras with straightening laws, in ring theory and algebra, Lect. Notes in Pure and Appl. Math. 55 (1980), 243-268.

[Elk87] Noam D Elkies, The existence of infinitely many supersingular primes for every elliptic curve over $\mathbb{Q}$, Inventiones mathematicae 89 (1987), no. 3, 561-567.

[Elk89]_, Supersingular primes for elliptic curves over real number fields, Compositio Mathematica 72 (1989), no. 2, 165-172.

[FW89] Richard Fedder and Keiichi Watanabe, A characterization of F-regularity in terms of F-purity, Commutative algebra (Berkeley, CA, 1987), Math. Sci. Res. Inst. Publ., vol. 15, Springer, New York, 1989, pp. 227-245. MR 1015520

[GW78] Shiro Goto and Keiichi Watanabe, On graded rings, $i$, Journal of the Mathematical Society of Japan 30 (1978), no. 2, 179-213.

[HP09] Christian Haase and Andreas Paffenholz, Quadratic Gröbner bases for smooth $3 \times 3$ transportation polytopes, Journal of Algebraic Combinatorics 30 (2009), no. 4, 477-489.

[HW85] Takayuki Hibi and Keiichi Watanabe, Study of three-dimensional algebras with straightening laws which are Gorenstein domains. I, Hiroshima Math. J. 15 (1985), no. 1, 27-54. MR 790025

[IT17] Nathan Ilten and Charles Turo, Unobstructed Stanley-Reisner degenerations for dual quotient bundles on G(2, n), Journal of Pure and Applied Algebra 221 (2017), no. 1, 119-134.

[Kol18] Mitra Koley, On Hilbert functions of graded rings and on the F-rationality of Rees algebras, Ph.D. thesis, Chennai Mathematical Institute, 2018, available at https://libarchive.cmi.ac.in/theses/mitrakoley-math2018.pdf.

[KS95] Michael Kalkbrener and Bernd Sturmfels, Initial complexes of prime ideals, Advances in Mathematics 116 (1995), no. 2, 365-376.

[Shi12] Takafumi Shibuta, Gröbner bases of contraction ideals, Journal of Algebraic Combinatorics 36 (2012), no. 1, 1-19.

[Stu96] Bernd Sturmfels, Gröbner bases and convex polytopes, vol. 8, American Mathematical Soc., 1996.

[Var09] Matteo Varbaro, Gröbner deformations, connectedness and cohomological dimension, Journal of Algebra 322 (2009), no. 7, 2492-2507.

[Var19] C Connectivity of hyperplane sections of domains, Communications in Algebra 47 (2019), no. 6, 2540-2547.

[Wat83] K-i Watanabe, Rational singularities with $k^{*}$-action, 1983.

E-mail address: aconstant@zedat.fu-berlin.de

Institute of Mathematics, Freie Universität Berlin, Germany

E-mail address: denegri@dima.unige.it

Dipartimento di Matematica, Universitá di Genova, Italy

E-mail address: varbaro@dima.unige.it

Dipartimento di Matematica, Universitá di Genova, Italy 